\title{
Measure of roughness of paper using speckle
}

\author{
Abdiel Pino ${ }^{a}$, Josep Pladellorens ${ }^{a}$ \\ ${ }^{a}$ CD6 Optics and Optometry Department, Polytechnical University of Catalonia \\ Rambla de Sant Nebridi 10, Terrassa, Spain 08222
}

\begin{abstract}
Roughness of a paper surface is particularly important in paper and board destined to be printed. Surfaces are often coated and the amount of coating and method of application used depends on the roughness of the base paper. We present a method of measure of the roughness of the paper based in the analysis of speckle pattern on the surface. Images are captured by means of a simple configuration using a laser and a camera CCD. Then, we apply digital image processing using the co-occurrence matrix, so this method can be considered as a non-contact surface profiling method, that can be used online.
\end{abstract}

Keywords: Paper roughness, speckle, co-occurrence matrix

\section{INTRODUCTION}

New use for printed paper products and search for minimization of production expenses require more accurate paper surface characterization. At the moment, the quality control of paper product is performed in the laboratory causing a great delay in the control of the final quality of the product. Paper web runs at speed of $30 \mathrm{~m} / \mathrm{s}$ and a lag in process control results in a major loss, since the quality between distinct runs varies remarkably.

Roughness of paper surface is an important parameter in paper manufacturing. Surface roughness measurement is one of the central measurement problems in paper industry. Surface roughness affects the major property of paper printing resolution [1], [2]. In addition to roughness, printing quality consists also of several other factors such as formation, printing method and porosity. At the moment, air leak methods are standardized and employed in paper industry as roughness rating methods. Air leak rate between measured paper surface and a specified flat land is recorded by using specialized pneumatic devices under laboratory conditions. Such a measurement closely corresponds to the roughness of a surface, the greater the air leak the rougher the surface. The measured roughness is given in micrometers or milliliters per second according to Parker Print-Surf (PPS) and Bendtsen methods, respectively. Air leak methods are rather easy to apply to paper and give stable results, although they measure roughness indirectly, need laboratory conditions, and thus unsuitable for on-line use. To measure real topography of paper surface, it is scanned with mechanical or optical profilometers. These methods provide accurate information on surface topography, but also demand laboratory conditions.

In our work, the image formed by speckle in the paper surface is considered as a texture, and therefore texture analysis methods are suitable for the characterization of paper surface. The results are contrasted to air leak methods.

\subsection{Paper surface roughness}

Paper surface topography is rated using smoothness or roughness. These two notations are complementary, smoothness meaning the degree to which surface is free from irregularities and inequalities and roughness meaning degree of unevenness or irregularity over the surface. The relation between paper surface roughness and internal pore structure of paper cannot be defined in theory. Paper surface consists of fibber grid, where the uppermost layers are not properly connected to lower layers. Roughness is an important factor in the final printing quality in printing papers, graphical boards, and many packaging boards [3]. The optical properties as gloss, the absorption of ink, and the amount of coating depend remarkably on surface roughness. A rough base paper needs more coating to cover the surface variation and in consequence is so important to know the surface roughness. Average roughness Ra is the area between the roughness profile and its mean line, or the integral of the absolute value of the roughness profile height over the evaluation length

$$
R a=\frac{1}{L} \int_{0}^{L} z(x) d x
$$

Optical Inspection and Metrology for Non-Optics Industries, edited by Peisen S. Huang,

Toru Yoshizawa, Kevin G. Harding, Proc. of SPIE Vol. 7432, 74320E - (c) 2009

SPIE · CCC code: 0277-786X/09/\$18 - doi: 10.1117/12.825072

Proc. of SPIE Vol. 7432 74320E-1 
Where $\mathrm{L}$ is the length of profile and $\mathrm{z}(\mathrm{x})$ is the height absolute value from the reference profile in point $\mathrm{x}$ [4], [5].

\subsection{Definition of Roughness}

The paper surface is characterized with three different roughness classes [5]:

- Optical roughness at length scales $<1 \mu \mathrm{m}$

- Micro roughness at $1 \mu \mathrm{m}-100 \mu \mathrm{m}$

- Macro roughness at $0.1 \mathrm{~mm}-1 \mathrm{~mm}$.

Optical roughness is connected to the surface properties of individual pigment particles and pulp fibbers. Micro roughness consists of the shapes and positions of fibbers in the paper surface. Macro roughness derives from the paper formation. All these three roughness classes affect paper gloss, and micro and macro roughness also paper uniformity. Macro roughness is a dominant attribute in the printing and coating properties of paper. A direct measurement of surface roughness is difficult. Roughness is usually defined as a deviation from an ideal, flat reference plane, where all the surface elements are in the same level [6]. The instruments for surface roughness characterization measure typically the deviation from a flat surface or compare the inspected surface to a reference one.

\subsection{Measuring paper surface roughness}

Methods that describe surface roughness are categorized to air leak, optical and profilometry methods. Each of these methods provides numerical roughness values from the measured surfaces, but the values may characterize different type or scale of roughness. In the air leak methods, air leak between flat land and inspected surface is measured. A smoother surface adjusts better to a probe; therefore air leak is slower between the inspected surface and the probe edges. At the moment, paper industry applies air leak methods, since they are easy to use and provide results, which correlate well with each other.

In optical methods the surface roughness measurement is based on the interaction between light and paper surface. One of the optical methods uses the projection of fringes over the surface and calculates the surface roughness from the deformation of the fringes. Another approach in optical methods is to measure light scattering, a rougher surface scatters more light than a smoother one. Finally, laser speckle measurement which is the method we use in this work uses the properties of speckle to measure the roughness of the surface.

\subsection{Air Leak Methods}

At the moment, the surface roughness is standardized to be measured with air leak methods. The air leak rate between measured paper surface and a specified flat land is recorded using specialized pneumatic devices; see Figure 1 for illustration of the Parker Print Surf (PPS) method. The air leak methods (Parker Print Surf, Bendtsen) have been developed till 1970 and only small adjustments have been made since then. The methods differ in the pressure on which the measuring land is pressed to paper, measured quantity (time, volume), softness of flat land or measured area.

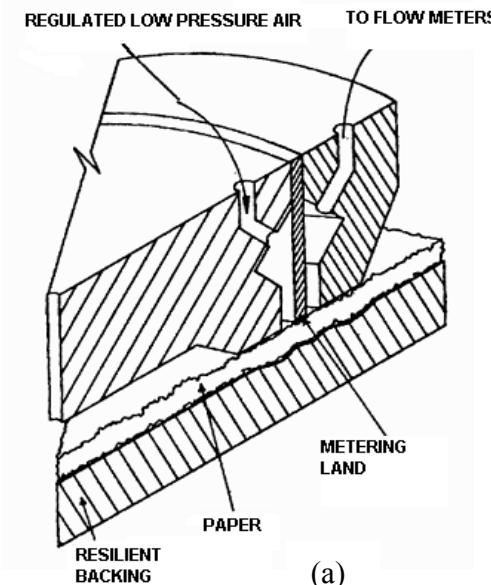

(a)

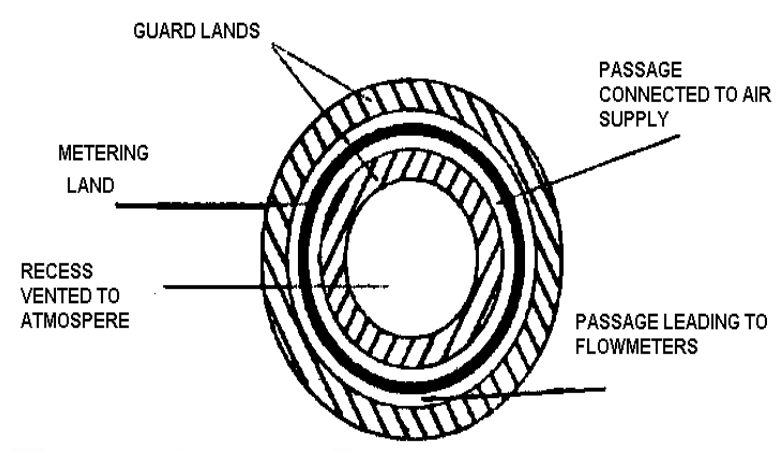

(b)

Figure 1: Parker Print Surf roughness head section. a) Seen from side b) seen from below. 


\subsection{Profilometers}

Profilometers measure the real topography of a surface. They have significantly better spatial resolution than air-leak devices. Profilometers are able to produce a 3D-image from the measured surface compared to a single value from the air-leak devices. The problem is that profilometers are too slow for on-line use. The basic idea of profilometers is that they have a stylus, which travels on a surface. Stylus is mechanical or optical. Mechanical stylus literally "sense" the surface by moving across the surface with a very sensitive probe. The spatial resolution is restricted to the stylus size, which is at smallest $10 \mu \mathrm{m}$.

Optical profilometers use laser as a stylus. In autofocusing instruments, a detector measures reflected laser light focused on the examined surface, and the light reflected from the surface is analysed by a detector, which observes deviations from the ideal focus position, the advantages to mechanical stylus are non-contact measurement and better resolution. The speed is still a problem with optical profilometers.

\subsection{Speckle}

Surface roughness measurement can be also accomplished by a speckle based instrument. A surface speckle pattern, which is a grainy structure in the space produced by scattered light from a rough surface when illuminated by coherent light, contains rich information about the surface fine geometrical properties, such as the surface roughness [7]. The speckle pattern images taken by an image sensor present texture form. The surface roughness information immersed in the speckle pattern images may be extracted by texture analysis.

Since the invention of lasers, researchers have discussed the relationships between surface roughness and speckle pattern statistical properties as a new method for off-line as well as on-line surface measurements. Using different properties of speckle fields and the different setup of optical systems have also developed a variety of speckle methods for surface roughness measurements [8], [9], [10]. For instance, surface roughness measurements may be implemented by the speckle pattern illumination methods, the speckle contrast methods, and the speckle correlation methods. Surface roughness measurement by means of speckle pattern illumination is convenient to determine roughness in the submicrometer range but requires a complicated optical illumination system consisting of a diffuser and a lens. The speckle contrast methods [11], [12], [13], [14], which are based on the first-order statistics of surface speckle patterns, can usually evaluate surface roughness values less than $R a<0.3 \mu \mathrm{m}$.

Speckle correlation methods [15], [16], [17], [18], which are based on second-order statistics, may work on surface roughness $R a$ between 1 and $30 \mu \mathrm{m}$. However, they often need two speckle pattern images, which may be obtained by one of the following methods: changing the incident light angle from the surface, rotating the surface to be measured, using two laser light beams. Such correlation methods are difficult to use for the in-process surface roughness measurement of moving objects, except for using two-laser light illumination.

Surface roughness extraction by means of texture analysis has been explored by some researchers [19], [20]. The surface roughness characterization method using the gray level co-occurrence matrix of surface texture images, which are captured by a vision system, has been investigated [21], [22], [23]. However, very little literature about surface roughness characterization, directly from speckle pattern texture images using texture analysis, can be found.

In this work, we will investigate the statistical properties of speckle pattern texture on the surface of paper from the point of view of computer texture analysis and present a simple method for characterizing a paper surface roughness from a single laser speckle pattern image, which is taken by a simple configuration setup consisting of a laser, beam expander and a CCD camera. The surface roughness is extracted by using the gray-level co-occurrence matrix (GLCM) of the speckle pattern texture image and quantized by the features of the GLCM.

\section{METODOLOGY}

\subsection{Theory and System Setup Configuration}

The basic configuration of the setup for surface roughness measurements by means of speckle pattern images are shown in Figure.2. The setup is built with a CCD camera UNIQ UM-301 with effective pixels 752 x 582 and a $5 \mathrm{~mW} \mathrm{He}-\mathrm{Ne}$ laser with a wavelength of $632.8 \mathrm{~nm}$, the power of which can be adjusted to avoid the digital camera signal saturation. The camera is located in the sample normal direction. The angle between the incident laser light beam and the normal direction is fixed to be as small as practically possible to reduce the effect of the direction of surface microstructure in 
the surface roughness evaluation. In the setup, the angle is $11^{\circ}$. By means of the simple setup, different speckle pattern images from paper surface roughness samples are obtained.

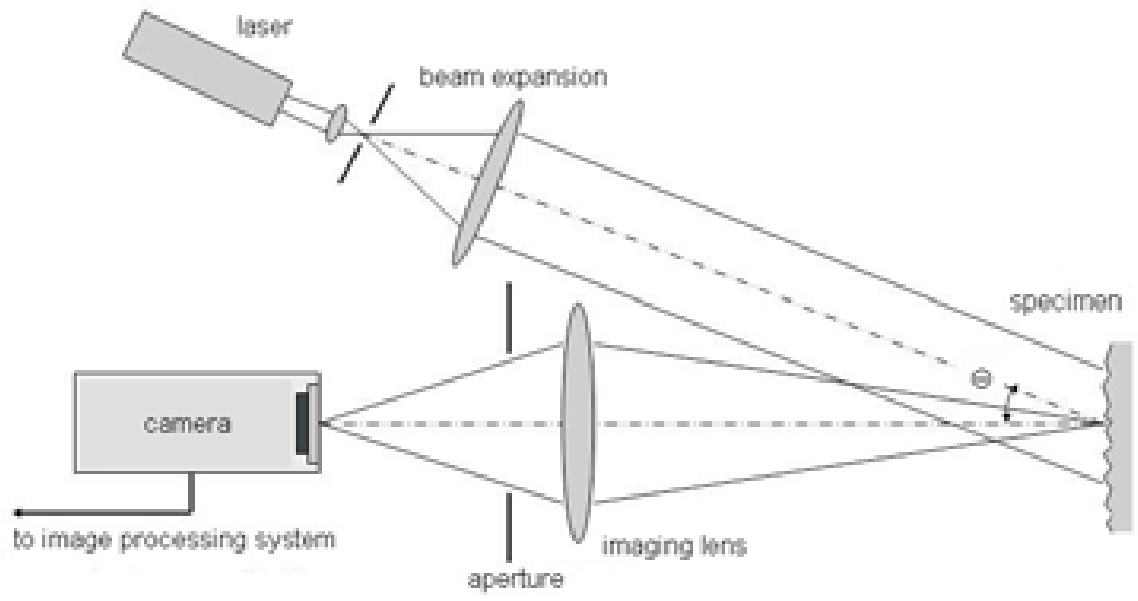

Figure. 2. The basic configuration of the setup for surface roughness measurements
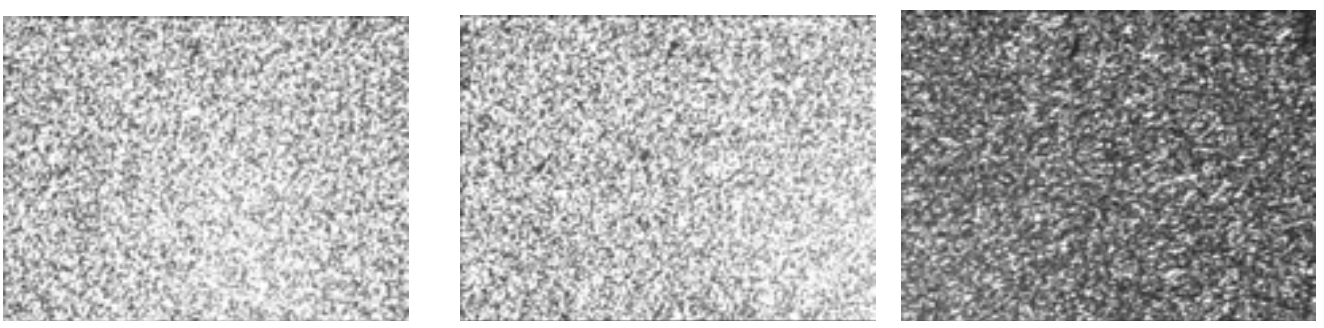

Figure. 3. Speckle pattern variations against the paper surface roughness.

From left to right of the images, it is apparent that the speckle pattern contrast becomes weaker and the image signal approaches saturation with the surface roughness $R a$ increasing. This implies that he speckle pattern texture properties change with the surface roughness, and it is possible to extract the surface roughness from the speckle pattern texture images using texture analysis.

\subsection{Computation of the Speckle Pattern Texture}

Information extraction from texture images can be obtained by different texture analysis methods, which are classified into four categories: statistical methods, geometrical methods, model-based methods, and signal processing methods. The gray-level co-occurrence matrix is based on second-order statistics, which deal with the spatial relationships of pairs of gray values of pixels in texture images. The texture image gray-level co-occurrence matrix indicates how often pairs of gray levels of pixels, which are separated by a certain distance $\mathrm{d}$ and lie along a certain direction $\theta$, occur in a texture image, as defined in [24].

\subsection{Co-occurrence matrix}

To calculate the co-occurrence matrix we consider (Figure 3) a direction and an offset. For a given direction and offset we determine the number of times gray level is repeated, this value normalize is the value of the cell of the co-occurrence matrix. In Figures 4 and 5 we show a test image with five gray tones and the calculation of the co-occurrence matrix for this test image. 


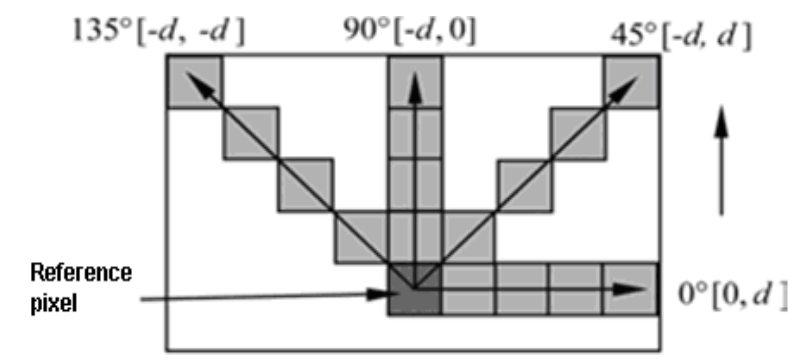

Figure. 3. Co-occurrence matrix offset and its direction definition.

\begin{tabular}{|l|l|l|l|l|}
\hline 4 & 4 & 3 & 0 & 0 \\
\hline 0 & 0 & 0 & 0 & 0 \\
\hline 1 & 0 & 0 & 2 & 2 \\
\hline 1 & 0 & 0 & 2 & 2 \\
\hline 1 & 1 & 3 & 2 & 2 \\
\hline
\end{tabular}

Figure 4. Test image with 5 gray tones

\begin{tabular}{|c|c|c|c|c|c|}
\hline Tone & 0 & 1 & 2 & 3 & 4 \\
\hline 0 & $\begin{array}{l}\text { Number } \\
(0,0)=7\end{array}$ & $\begin{array}{l}\text { Number } \\
(0,1)=0\end{array}$ & $\begin{array}{l}\text { Number } \\
(0,2)=2\end{array}$ & $\begin{array}{l}\text { Number } \\
(0,3)=0\end{array}$ & $\begin{array}{l}\text { Number } \\
(0,4)=0\end{array}$ \\
\hline 1 & $\begin{array}{l}\text { Number } \\
(1,0)=2\end{array}$ & $\begin{array}{l}\text { Number } \\
(1,1)=1\end{array}$ & $\begin{array}{l}\text { Number } \\
(1,2)=0\end{array}$ & $\begin{array}{l}\text { Number } \\
(1,3)=1\end{array}$ & $\begin{array}{l}\text { Number } \\
(1,4)=0\end{array}$ \\
\hline & $\begin{array}{l}\text { Number } \\
(2,0)=0\end{array}$ & $\begin{array}{l}\text { Number } \\
(2,1)=0\end{array}$ & $\begin{array}{l}\text { Number } \\
(2,2)=3\end{array}$ & $\begin{array}{l}\text { Number } \\
(2,3)=0\end{array}$ & $\begin{array}{l}\text { Number } \\
(2,4)=0\end{array}$ \\
\hline & $\begin{array}{l}\text { Number } \\
(3,0)=1\end{array}$ & $\begin{array}{l}\text { Number } \\
(3,1)=0\end{array}$ & $\begin{array}{l}\text { Number } \\
(3,2)=1\end{array}$ & $\begin{array}{l}\text { Number } \\
(3,3)=0\end{array}$ & $\begin{array}{l}\text { Number } \\
(3,4)=0\end{array}$ \\
\hline & $\begin{array}{l}\text { Number } \\
(4,0)=0\end{array}$ & $\begin{array}{l}\text { Number } \\
(4,1)=0\end{array}$ & $\begin{array}{l}\text { Number } \\
(4,2)=0\end{array}$ & $\begin{array}{l}\text { Number } \\
(4,3)=1\end{array}$ & $\begin{array}{l}\text { Number } \\
(4,4)=1\end{array}$ \\
\hline
\end{tabular}

Figure 5. Co-occurrence matrix for $0^{0}$ and $\mathrm{d}=1$, before normalization

A co-occurrence matrix is capable of texture analysis by using texture features extracted from gray-level co-occurrence matrix GLCM (equation 2). Haralick [24], [25] suggested 14 textural features that can be used. Here, we chose four features, contrast, correlation, energy, and homogeneity, for speckle pattern texture analysis. The contrast (equation 3) feature measures the local variations in the gray-level co-occurrence matrix; correlation (equation 4) measures the joint probability occurrence of the specified pixel pairs; energy (equation 5), which is also known as uniformity or the angular second moment, provides the sum of squared elements in the GLCM; and homogeneity (equation 6) measures the closeness of the distribution of elements in the GLCM to the GLCM diagonal.:

$$
\begin{gathered}
P_{i, j}=\frac{C_{i, j}}{\sum_{i, j=0}^{N-1} C_{i, j}} \\
\sum_{i, j=0}^{N-1} P_{i, j}(i-j)^{2} \\
\sum_{i, j=0}^{N-1}\left[\frac{\left(i-\mu_{i}\right)\left(j-\mu_{j}\right)}{\sqrt{\left(\sigma_{i}^{2}\right)\left(\sigma_{j}^{2}\right)}}\right] \\
\sum_{i, j=0}^{N-1} P_{i, j}^{2} \\
\sum_{i j,=0}^{N-1} \frac{P_{i, j}}{1+(i-j)^{2}}
\end{gathered}
$$




\section{RESULTS}

We have used the setup of figure 2 . To test the method of the texture analysis using the matrix of co-occurrence we have used the energy descriptor of the matrix of co-occurrence obtained from images of speckle pattern over the surface of the paper we have use two handmade papers with fibers of eucalyptus and pine. In figures 6 and 7 we have plotted the energy descriptor of the matrix of co-occurrence with respect the offset for the four directions $\left(0^{\circ}, 45^{\circ}, 90^{\circ}\right.$ and $\left.135^{\circ}\right)$, corresponding to the pine and eucalyptus sheets. The slopes of the lines that fit the data have a mean value equal to -0.00339 for the pine sheet and to eucalyptus sheet -0.00171 . In table 1 we show the results obtained in twelve tests for the pine and eucalyptus sheets. The mean value of the slopes in the two sheets shows a difference that corresponds to the difference of the Bendtsen test for the two sheets.
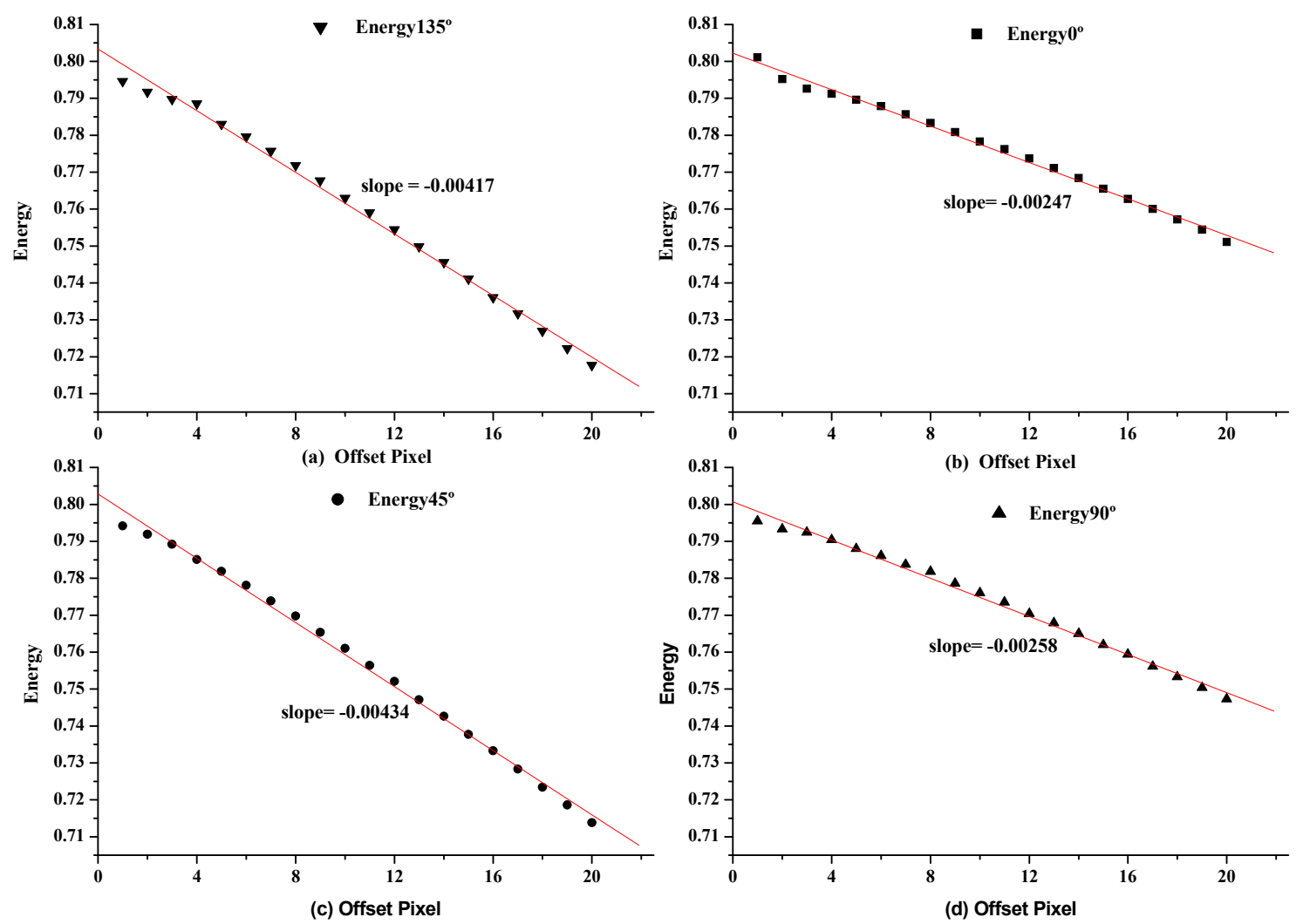

Figure 6. Plots of the energy of the matrix co-occurrence with respect the offset for the four directions $\left(0^{\circ}, 45^{\circ}, 90^{\circ}\right.$ and $135^{\circ}$ ), corresponding to the pine sheet. The slopes of the lines that fit the data have a mean value equal to -0.00339 . 

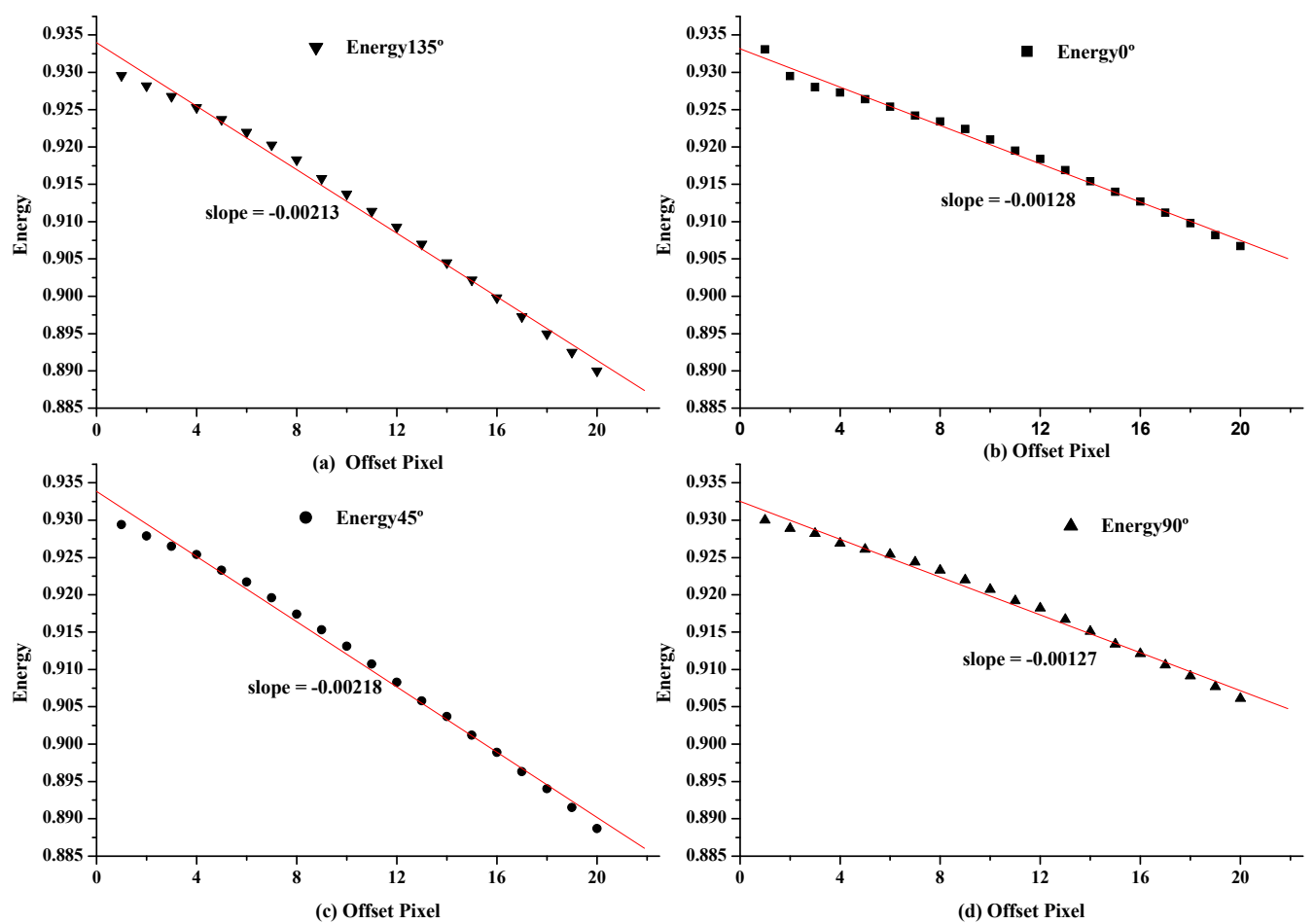

Figure 7. Plots of the energy of the matrix co-occurrence with respect the offset for the four directions $\left(0^{\circ}, 45^{\circ}, 90^{\circ}\right.$ and $135^{\circ}$ ), corresponding to the eucalyptus sheet. The slopes of the lines that fit the data have a mean value equal to 0.00171 .

Table. 1. Values of the Bendtsen parameter for the samples of eucalyptus and pine papers sheets in twelve tests with the mean value.

\begin{tabular}{|c|c|c|}
\hline \multicolumn{3}{|c|}{ Smoothness Bendtsen (mL/min) } \\
\hline & Eucalyptus & Pine \\
\hline 1 & 600 & 1300 \\
\hline 2 & 700 & 1100 \\
\hline 3 & 600 & 1200 \\
\hline 4 & 700 & 1000 \\
\hline 5 & 700 & 1100 \\
\hline 6 & 700 & 1000 \\
\hline 7 & 700 & 1000 \\
\hline 8 & 600 & 1200 \\
\hline 9 & 700 & 1100 \\
\hline 10 & 600 & 1100 \\
\hline 11 & 700 & 1100 \\
\hline 12 & 700 & 1100 \\
\hline Mean & $\mathbf{6 6 7}$ & $\mathbf{1 1 0 8}$ \\
\hline
\end{tabular}




\section{CONCLUSIONS}

We have put forward a surface roughness measurement technique through investigating the features of the texture image gray-level co-occurrence matrix of the surface speckle pattern. There are many texture features that can be extracted from the gray-level co-occurrence matrix. In our research, the four commonly used features contrast, correlation, energy, and homogeneity are studied with respect to surface roughness. We have found that the variation of the energy feature with different offset establishes a clear difference between two papers that have very different values of the Bendtsen parameter, which measures the roughness of the paper through air leak. The work has just begin, we need to characterize more papers in order to probe the method and know what capabilities the system could have

\section{ACKNOWLEDGMENTS}

We would like to thank to Profs J.Colom and A.Torres of the Textile and Paper Department of the Polytechnical University of Catalonia and to the PhD student Miguel Ares for the help provided and also to Miquel y Costas \& Miquel S.A. for his financial support.

\section{REFERENCES}

${ }^{[1]}$ Corte, H. [The structure of paper. The structure and physical properties of paper], Elsevier, 157-282 (1982).

${ }^{[2]}$ Cresson, T. and Luner, P., "The characterization of paper formation. Part 2: The texture analysis of paper formation", Tappi Journal, 73(12),175-184 (1990).

${ }^{[3]}$ Rance, H.F., [Handbook of paper science, vol 2:The structure and physical properties of paper], Elsevier Scientific Publishing (1998).

${ }^{[4]}$ Rakels, J., "Recognised surface finish parameters obtained from diffraction patterns of rough surfaces," Proceedings of SPIE (1988).

${ }^{[5]}$ Kajanto, I., Laamanen, J. and Kainulainen, M., "Paper bulk and surface," Paper Physics. K. Niskanen. Helsinki, Fapet Oy. 16, 89-115 (1998).

${ }^{[6]}$ Whitehouse, D. J., [Handbook of Surface and Nanometrology]. Institute of Physics, (2003).

${ }^{[7]}$ Goodman, J. W., [Speckle Phenomena in Optics: Theory and Applications], Roberts \& Company Publishers, (2006).

${ }^{[8]}$ Asakura, T., "Surface roughness measurements," Speckle Metrology, R. K. Erf, ed. Academic New York, (1978).

${ }^{[9]}$ Dainty, J. C., "Laser Speckle and Related Phenomena", Vol. 9 Topics in Applied Physics Springer-Verlag, (1984).

${ }^{[10]}$ Pedersen, H. M. "Theory of speckle dependence on surface roughness," J. Opt. Soc. Am. 66, 1204-1210 (1976).

${ }^{[11]}$ Duncan, D. D., Kirkpatrick, S. J., and Wang, R. K., "Statistics of local speckle contrast," J. Opt. Soc. Am. A, 25(1) (2008).

${ }^{[12]}$ Briers, J. David, "Laser speckle contrast imaging for measuring blood flow," Proceedings of the Symposium on Photonics Technologies for 7th Framework Program Wroclaw, (2006).

${ }^{[13]}$ Cheng, C., Liu, C., Zhang, N., Jia, T., Li, R., Xu, Z. ,"Absolute measurement of roughness and lateral correlation length of random surfaces by use of the simplified model of image-speckle contrast," Appl. Opt. 41, 4148-4156 (2002).

${ }^{[14]}$ Fujii, H. and Asakura, T., "Measurement of surface roughness properties by using image speckle contrast", J. Opt. Soc. Am., 66(11) (1976).

${ }^{[15]}$ Chandley, P. J. and Escamilla, H. M., "Speckle from a rough surface when the illuminated region contains few correlation areas: the effect of changing the surface height variance," Opt. Commun. 29, 151-154 (1979).

${ }^{[16]}$ Léger, D., Perrin, J. C. , "Real-time measurement of surface roughness by correlation of speckle patterns," J. Opt. Soc. Am. 66 (11) (1976).

${ }^{[17]}$ Ruffing, B., "Application of speckle-correlation methods to surface-roughness measurement: a theoretical study", J. Opt. Soc. Am. A 3, 1297-1304 (1986).

${ }^{[18]}$ Fricke-Begemann, Thomas, Hinsch, Klaus D., "Measurement of random processes at rough surfaces with digital speckle correlation," J. Opt. Soc. Am. 21(2) (2004).

${ }^{[19]}$ Chang, S. I. and Ravathur, J. S., "Computer vision based on no-contact surface roughness assessment using wavelet transform and response surface methodology," Qual. Eng. 17, 435-451 (2005). 
${ }^{[20]}$ Kiran, M. B., Ramamoorthy, B., Radhakrishnan, V., “Evaluation of surface roughness by vision system,” Int. J. Mach. Tools Manuf. 38, 685-690 (1998).

${ }^{[21]}$ Latif-Amet, L., Ertuzun, A., and Ercil, A., "An efficient method for texture defect detection: Subband domain cooccurrence matrices,". Image and Vision Computing, 18(6), 543-553 (2000).

${ }^{[22]}$ Gadelmawla, E. S., "A vision system for surface roughness characterization using the gray level cooccurrence matrix," NDT \& E Int. 37, 577-588 (2004).

${ }^{[23]}$ Lee, C., Chao, Y. J., "Surface texture dependence on surface roughness by computer vision," in IEEE International Conference on Robotics and Automation 4, 520-524 (1987).

${ }^{[24]}$ Haralick, R. M., Shanmugam, K., Dinstein, I., “Texture features for image classification,” IEEE Trans.Syst. Man Cybern. 3, 610-621 (1973).

${ }^{[25]}$ Haralick, R., "Statistical and structural approaches to texture,". Proceedings of the IEEE, 67(5), 786-804 (1979). 